\title{
Bead-based kinase profiling
}

\section{By Michael J. Haas, Senior Writer}

A team of researchers from the Broad Institute and other institutions has developed a high throughput screening method to profile activated tyrosine kinases in cancer cells. ${ }^{1}$ The group applied the approach to glioblastoma multiforme and showed that the technology could help identify new cancer targets missed by genomics approaches.

Although the team also published kinase activation profiles for an extensive number of cancer cell lines, corporate researchers said more proof of principle will be required to make effective use of the technology.

Activated (phosphorylated) tyrosine kinases play a central role in oncogenesis. But current gene resequencing methods for identifying mutations associated with cancer cannot directly identify enzymes with aberrant phosphorylation patterns that are independent of

"This is the newest and perhaps best technology for an initial survey of many of the relevant tyrosine kinases for activation levels in one shot."

- David Hangauer,

Kinex Pharmaceuticals LLC

\section{Story SRC}

"This is the newest and perhaps best technology for an initial survey of many of the relevant tyrosine kinases for activation levels in one shot," said David Hangauer, SVP of R\&D at Kinex Pharmaceuticals LLC.

Kinex's KX2-391 selective v-src sarcoma (Schmidt-Ruppin A-2) viral oncogene homolog (avian) (SRC) inhibitor is in a Phase I trial to treat solid tumors.

Hangauer, who is also an associate professor of medicinal chemistry and chemistry at The State University of New York at Buffalo, did note that the technique could only reveal which kinases were phosphorylated in a particular cancer cell line-not which proteins those kinases might themselves subsequently phosphorylate.

Thus, he suggested the approach would be most useful for finding inhibitors of kinase activation but said the technique published in Nature Biotechnology was one of several that Kinex would want to use in its own efforts to discover inhibitors of already activated kinases.

Dennis France, VP of molecular oncology at ArQule Inc., said the phosphorylation signatures for dozens of cell lines provided in the Nature Biotechnology paper could help identify new cancer targets. But he added that such results would be more useful to conventional kinase hunters than to ArQule, which uses its ArQule kinase inhibitor discovery platform (AKIP) to design molecules that target therapeutically relevant kinases in their inactive conformations.

France was more interested in the tumor biology data presented in the paper's supplementary

The team, led by Todd Golub, director of the cancer program at the Broad Institute of Massachusetts Institute of Technology (MIT) and Harvard University, thus set out to use phosphorylation status as a direct indicator of whether a specific tyrosine kinase might play a role in a given cancer type.

The researchers validated antibodies against 62 of the approximately 90 known tyrosine kinases in the human genome and then coupled the antibodies to color-coded polystyrene beads, with one kinase per bead. They then exposed the bead library to a lysate from a cancer cell line or a patient sample to isolate all expressed tyrosine kinases.

A key step was the subsequent addition of a second labeled antibody that would specifically bind to phosphotyrosine residues. This enabled the team to use Luminex Corp.'s xMAP flow cytometry technology to detect phosphorylated tyrosine kinases and use the results to generate cell line-specific and sample-specific profiles of these 62 activated tyrosine kinases.

For its report, published in Nature Biotechnology, the team profiled 130 human cancer cell lines and presented the kinase activation profiles for each in the paper's supplementary information.

Golub, who was not available for comment, is also an associate professor of pediatrics at Harvard Medical School and an investigator at both the Dana-Farber Cancer Institute and the Howard Hughes Medical Institute. information. "Knowing that cell line ' $\mathrm{X}$ ' has activated receptor tyrosine kinase ' $\mathrm{Y}$ ' is news you can use," he said.

After identifying a lead compound, "we spend lots of time scouting appropriate cells lines for the compound's target, determining whether it is overexpressed or activated and whether the compound knocks it down," France said.

This labor-intensive process could be streamlined by the data provided in Nature Biotechnology. "I will definitely be mining these data for the sweet spots in our programs," France told SciBX. "If we had the money in the budget, we would get this technology right now to generate a tumor biology database. If our platform really takes off, then we will probably get this technology later on, especially if it were validated a bit further."

ArQule's lead compound is ARQ197, a small molecule inhibitor of c-Met receptor tyrosine kinase (MET). It is in Phase II trials to treat pancreatic cancer, non-small cell lung cancer (NSCLC) and microphthalmia transcription factor (MITF) tumors. Last month, the company partnered with Daiichi Sankyo Co. Ltd. to co-develop ARQ197 in the U.S., EU, South America and the rest of the world. The deal excludes Japan, China, South Korea and Taiwan, where Kyowa Hakko Kirin Co. Ltd. holds rights.

John Lyons, VP of translational research at Astex Therapeutics Ltd., said, "The technology is a very powerful addition to the sequencing and 
gene and protein expression arrays now in vogue" and eventually might replace Western blotting as the method of choice for profiling kinases.

"An advantage of the method might be compound profiling and comparing a number of lead compounds in a drug discovery program or perhaps comparing specificity of competitor compounds in achieving complete cellular responses," he said.

Neil Thompson, SVP of biology at Astex, agreed, noting that the technique had a higher throughput and required less sample than other methods for profiling kinases, such as mass spectrometry. "This will make it useful for comparison studies of cells, compounds and tissues," he said.

But Thompson noted that the technique had at least two limitations: it required additional experiments to determine whether the phosphorylation of a given tyrosine residue has any physiological (disease) relevance and it depended heavily on the specificity of the anti-tyrosine kinase antibodies.

Astex has AT9283, an inhibitor of aurora kinase A (AURKA), AURKB, BCR-ABL tyrosine kinase and Janus kinase 2 (JAK2), in a Phase I/II trial to treat acute myeloid leukemia (AML), chronic myeloid leukemia (CML), acute lymphoblastic leukemia (ALL), myelodysplastic syndrome (MDS) and myelofibrosis.

Thompson also said it wasn't clear from the paper how much quality control of antibodies was necessary in the study. "This type of thing will have a major impact on how useful it proves to be in other laboratories with less experience in the process," he said.

Jinyan Du, lead author on the paper, told SciBX that the team obtained antibodies against approximately 80 tyrosine kinases but only 62 could be validated for specificity for use in the team's study. Du is a research fellow at both Dana-Farber and the Broad Institute.

\section{Confirmation in GBM}

To show that the technique can find cancer targets that are overlooked by genomics screens, the authors analyzed primary glioblastoma multiforme (GBM) tumor samples from human patients. They zeroed in on SRC, a known cancer target whose role in disease does not involve mutation, and proceeded to show that a marketed SRC inhibitor-Sprycel dasatinib-blocked GBM cell migration in vitro and slowed GBM tumor growth in mice.

Thus, the authors wrote, their results demonstrated that the screening method could identify a potential target to treat GBM-SRC, which genomics studies had not previously linked to cancer. ${ }^{2}$

ArQule's France thought the follow-on experiments provided sufficient proof of concept to make the method attractive but said he wanted to see more pharmacological data in multiple cell lines-not just SRC inhibition in GBM.

"For instance," he said, "they could have picked a few drug compounds with known targets-such as Sutent or Gleevec-and shown whether these actually knock down the respective target receptor tyrosine kinases that lit up" in the cell lines profiled.

France also said follow-on experiments in a lesser known kinase than
SRC might have been more illustrative of the technique's potential. As an example, he pointed out that the team's results showed that AXL receptor tyrosine kinase (AXL; UFO) was activated in many cancer cell lines.

Compared with SRC, he said, AXL is a new target and there are no known inhibitors. An exploration of the observed AXL correlations might have demonstrated that the technique "could be used to identify receptor tyrosine kinases that have not received much attention to date," he said.

Going forward, Du said, the researchers are expanding the technique to include all known tyrosine kinases as well as some downstream signaling molecules.

She said the team is also examining the exact role SRC plays in GBM. "We are also interested to see whether dasatinib could produce synergistic effects with other chemotherapy drugs" in GBM, she said.

Du said the team has no plans to apply for a patent. "We would like this technique to become widely available to the community," she said.

In addition to Broad, Harvard Medical, Dana-Farber and Howard Hughes, the research team included scientists from Harvard University, Massachusetts General Hospital, the David Geffen School of Medicine at the University of California, Los Angeles and Memorial Sloan-Kettering Cancer Center.

Bristol-Myers Squibb Co. markets Sprycel, an inhibitor of BCR-ABL and SRC, to treat ALL and CML. Pfizer Inc. markets Sutent sunitinib, an inhibitor of multiple tyrosine kinases, to treat gastrointestinal stromal tumors (GIST) and renal cell carcinoma (RCC). Novartis AG markets Gleevec imatinib, a BCR-ABL inhibitor, to treat CML, ALL, MDS and GIST.

Haas, M.J. SciBX 2(3); doi:10.1038/scibx.2009.82

Published online Jan. 22, 2009

\section{REFERENCES}

1. Du, J. et al. Nat. Biotechnol.; published online Dec. 21, 2008; doi:10.1038/nbt.1513

Contact: Todd R. Golub, Broad Institute, Cambridge, Mass. e-mail: golub@broad.mit.edu

2. Cancer Genome Atlas Research Network. Nature 455, 1061-1068 (2008)

COMPANIES AND INSTITUTIONS MENTIONED

ArQule Inc. (NASDAQ:ARQL), Woburn, Mass.

Astex Therapeutics Ltd., Cambridge, U.K.

Bristol-Myers Squibb Co. (NYSE:BMY), New York, N.Y.

Broad Institute, Cambridge, Mass.

Daiichi Sankyo Co. Ltd. (Tokyo:4568; Osaka:4568), Tokyo, Japan

Dana-Farber Cancer Institute, Boston, Mass.

Harvard Medical School, Boston, Mass.

Harvard University, Boston, Mass.

Howard Hughes Medical Institute, Chevy Chase, Md.

Kinex Pharmaceuticals LLC, Buffalo, N.Y.

Kyowa Hakko Kirin Co. Ltd. (Tokyo:4151), Tokyo, Japan

Luminex Corp. (NASDAQ:LMNX), Austin, Texas

Massachusetts General Hospital, Boston, Mass.

Massachusetts Institute of Technology, Cambridge, Mass.

Memorial Sloan-Kettering Cancer Center, New York, N.Y.

Novartis AG (NYSE:NVS; SWX:NOVN), Basel, Switzerland

Pfizer Inc. (NYSE:PFE), New York, N.Y.

The State University of New York at Buffalo, Buffalo, N.Y.

University of California, Los Angeles, Calif. 